\title{
Tinkering under the Hood: Metabolic Optimisation of CAR-T Cell Therapy
}

\author{
Yasmin Jenkins ${ }^{1}$, Joanna Zabkiewicz ${ }^{2}$, Oliver Ottmann ${ }^{2}$ and Nicholas Jones ${ }^{1, *}$ \\ 1 Institute of Life Science, Swansea University Medical School, Swansea University, Swansea SA2 8PP, UK; \\ 952343@swansea.ac.uk \\ 2 Experimental Cancer Medicine Center, Department of Haematology, Heath Hospital, Cardiff University, \\ Heath Park, Cardiff CF14 4XN, UK; zabkiewiczj1@cardiff.ac.uk (J.Z.); OttmannO@cardiff.ac.uk (O.O.) \\ * Correspondence: n.jones@swansea.ac.uk
}

Citation: Jenkins, Y.; Zabkiewicz, J.; Ottmann, O.; Jones, N. Tinkering under the Hood: Metabolic Optimisation of CAR-T Cell Therapy. Antibodies 2021, 10, 17. https:// doi.org/10.3390/antib10020017

Academic Editor: Christian Klein

Received: 10 February 2021

Accepted: 20 April 2021

Published: 26 April 2021

Publisher's Note: MDPI stays neutral with regard to jurisdictional claims in published maps and institutional affiliations.

Copyright: (c) 2021 by the authors. Licensee MDPI, Basel, Switzerland. This article is an open access article distributed under the terms and conditions of the Creative Commons Attribution (CC BY) license (https:/ / creativecommons.org/licenses/by/ $4.0 /)$.

\begin{abstract}
Chimeric antigen receptor (CAR)-T cells are one of the most exciting areas of immunotherapy to date. Clinically available CAR-T cells are used to treat advanced haematological B-cell malignancies with complete remission achieved at around 30-40\%. Unfortunately, CAR-T cell success rates are even less impressive when considering a solid tumour. Reasons for this include the paucity of tumour specific targets and greater degree of co-expression on normal tissues. However, there is accumulating evidence that considerable competition for nutrients such as carbohydrates and amino acids within the tumour microenvironment (TME) coupled with immunosuppression result in mitochondrial dysfunction, exhaustion, and subsequent CAR-T cell depletion. In this review, we will examine research avenues being pursued to dissect the various mechanisms contributing to the immunosuppressive TME and outline in vitro strategies currently under investigation that focus on boosting the metabolic program of CAR-T cells as a mechanism to overcome the immunosuppressive TME. Various in vitro and in vivo techniques boost oxidative phosphorylation and mitochondrial fitness in CAR-T cells, resulting in an enhanced central memory $\mathrm{T}$ cell compartment and increased anti-tumoural immunity. These include intracellular metabolic enhancers and extracellular in vitro culture optimisation pre-infusion. It is likely that the next generation of CAR-T products will incorporate these elements of metabolic manipulation in CAR-T cell design and manufacture. Given the importance of immunometabolism and $\mathrm{T}$ cell function, it is critical that we identify ways to metabolically armour CAR-T cells to overcome the hostile TME and increase clinical efficacy.
\end{abstract}

Keywords: CAR-T cells; metabolism; immunometabolism; oncometabolism; tumour microenvironment

\section{Introduction}

Chimeric antigen receptor (CAR)-T cells are currently at the forefront of cancer immunotherapy [1] and are one of the most promising treatments for advanced haematological malignancies [2,3]. CAR-T cells are genetically engineered $\mathrm{T}$ cells that express an artificial receptor targeting a tumour specific antigen. The generation and expansion of CAR-T cells involves either removing the patient's autologous T cells or using healthy donor T cells (allogeneic), applying genetic modification of the T cells to express the CAR construct, and finally expanding the CAR-T cells prior to patient infusion [3,4]. During the genetic modification (via (retro)viral vector mediated delivery or other means) and expansion process, CAR-T cells are cultured ex vivo in media formulations that contain excessive, non-physiological nutrient levels [4]. Whilst the utilisation of media formulations with excessive nutrients are critical for successful CAR-T cell expansion to generate sufficient numbers for patient infusion, heightened nutrient levels may compromise the function and survival of CAR-T cells in vivo when they encounter a more hostile, nutrient-restrictive environment [5]. These metabolic challenges within the tumour microenvironment (TME) include glucose and amino acid restrictions, reduced oxygen tensions, and an abundance of immunosuppressive nutrients such as lactate and kynurenine [5-7]. Aside from the TME, 
metabolic challenges can also arise in the pre-infused CAR-T cell product, as autologous donor $\mathrm{T}$ cells are often collected post-treatment (chemotherapy, radiotherapy, etc.) that can inflict long-lasting metabolic defects such as mitochondrial damage [8]. Furthermore, the aforementioned treatments are accompanied with different degrees of aggressivity that is dependent on age [8]. Whilst, previous strategies for enhancing CAR-T cell therapy have focussed on enhancing T cell receptor (TCR)-related and co-stimulatory pathways, recently consideration is being given to optimising nutrient culturing conditions and strategically enhancing metabolism to better equip CAR-T cells in the TME [9].

To date, CAR-T cells have been considered a success in the treatment of haematological cancers such as B cell acute lymphoblastic leukaemia and B cell lymphomas, however, success rates are less impressive for the treatment of solid tumours [10]. Metabolic manipulation could overcome the barriers of CAR-T cell success in solid tumours and certainly warrants further investigation.

The renaissance of immunometabolism as an area of research dictates the metabolic pathways that govern immune cell fate and function [11]. Metabolic programming in T cells has come under recent scrutiny, revealing key roles in human health and disease. We now know that metabolic heterogeneity is demonstrated by the broad range of $\mathrm{T}$ cell compartments. For example, quiescent $\mathrm{T}$ cells (e.g., naïve and resting memory $\mathrm{T}$ cells) rely on catabolic processes such as oxidative phosphorylation (OXPHOS) to maintain housekeeping functions [12], whereas upon activation, T cells (e.g., early activated and effector T cells [13]) shift to anabolic pathways such as glycolysis (inclusive of horizontal pathways such as the pentose phosphate pathway and serine biosynthesis pathway) and the tricarboxylic cycle to generate biosynthetic intermediates required for proliferation $[13,14]$.

This mini-review will discuss our current understanding of $\mathrm{T}$ cell metabolism and how this may impact the design and culture of CAR-T cells for therapeutic use. We will explore how metabolism can shape CAR-T cell memory, persistence, and effector function, and give considerations into how CAR-T cell metabolism can be modulated to optimise the efficacy of CAR-T cell therapy in the setting of solid tumours.

\section{T Cell Metabolism and the Tumour Microenvironment}

Throughout their life cycle, $\mathrm{T}$ cells encounter a range of energetically challenging microenvironments within the human body and are therefore challenged with the need to adapt to an altered nutrient environment dictated by cytokine/chemokine milieu and cell-cell competition in order to provide host protection [15]. All too frequently, the hostile TME [16] results in the functional impairment of immune effector cells by manipulating the metabolic programs of anti-tumour $\mathrm{T}$ cell populations via numerous mechanisms. One such mechanism is the direct competition for nutrients [7]. To maintain their highly energetic phenotype, cancer cells are often/typically intrinsically programmed to transport large quantities of glucose (to support aerobic glycolysis, or the 'Warburg-effect') and amino acids to support their exponential proliferation, thereby outcompeting local immune cell populations, including intratumoural T cells [17].

Elevated levels of aerobic glycolysis by the tumour results in localised acidosis via extensive lactate production [18]. Once considered a waste product, lactate has now been shown to have both intrinsic and extrinsic effects on T cell function (Figure 1). For example, in lymph nodes, elevated lactate levels driven by low convective flow and heightened glucose metabolism inhibit $\mathrm{T}$ cell function to circumvent an autoimmune reaction [19]. Such parallels can be drawn between the lymph node microenvironment and TME, whereby lactate inhibits the effector T cell function [20]. Excess lactate impairs NAD+ regeneration, subsequently blocking the enzymatic reactions involving glyceraldehyde 3-phosphate dehydrogenase and 3-phosphoglycerate dehydrogenase [21]. This blockade reduces $\mathrm{T}$ cell proliferation, but can be rescued by restoration of the redox balance via supplementation with serine [21]. Furthermore, within the TME, lactate can promote the differentiation of naïve $\mathrm{T}$ cells into regulatory $\mathrm{T}$ cells (Tregs) whereby the carbon backbone directly enters the citric acid (TCA) cycle and contributes to energy production via OXPHOS. The ability 
of Tregs to thrive in a low-glucose, high-lactate TME enhances immunosuppression and tumour evasion [22] and contributes to the progression of malignancy [22]. In addition, lactate impacts on natural killer (NK) cell viability by promoting mitochondrial dysfunction leading to apoptosis [18]. Given the multiple negative implications of lactate produced by multiple cell types on anti-tumour immunity, the metabolic targeting of aerobic glycolysis is potentially an attractive therapeutic target. Indeed, the restriction of tumour glycolysis levels using the non-steroidal anti-inflammatory drug diclofenac has been demonstrated to reduce lactate levels and augment immune checkpoint therapy via the preservation of the effector T cell function [23].

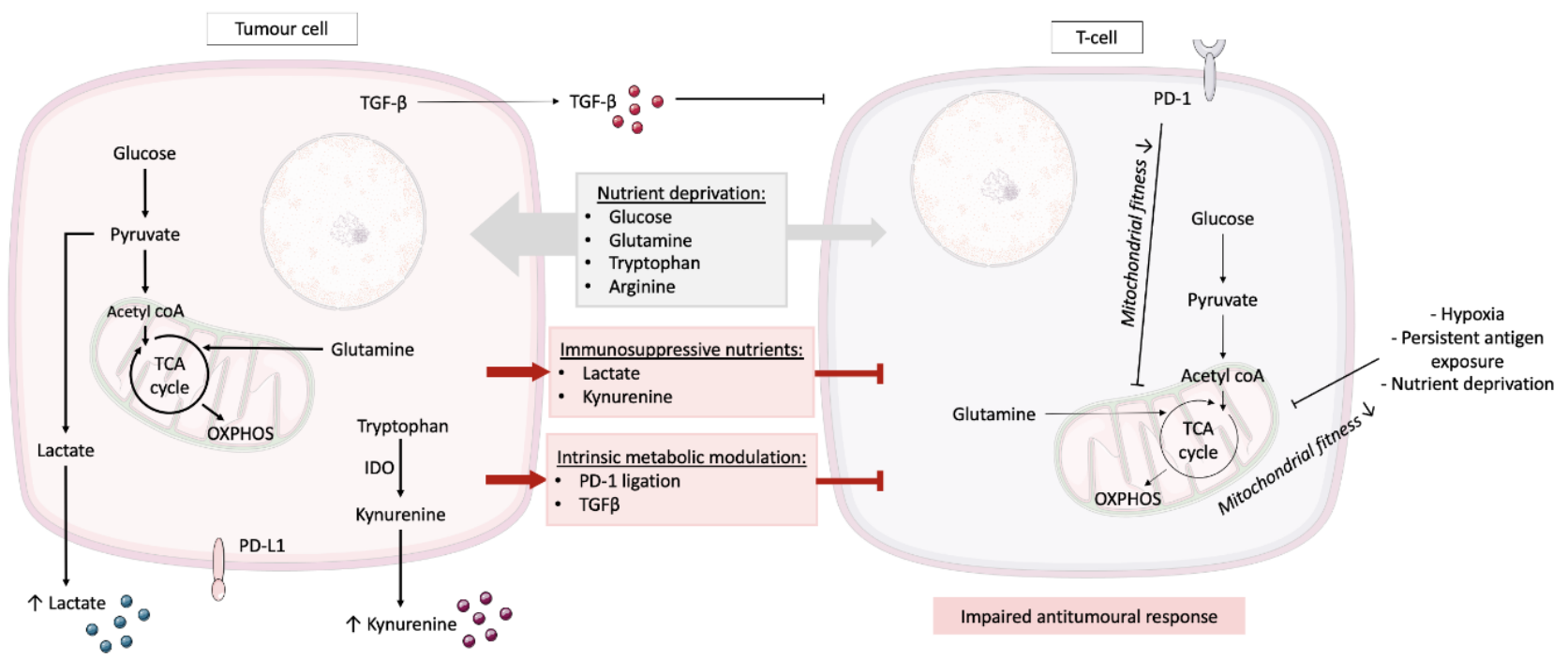

Figure 1. T cell metabolism and the tumour microenvironment. T cells face various metabolic challenges within the TME. Direct competition for nutrients arises such as glucose, glutamine, and amino acids, with the tumour outcompeting $T$ cells. The tumour can also produce immunosuppressive nutrients such as lactate and kynurenine, and intrinsically modulate T cell metabolism through PD- 1 checkpoint ligation and the production of TGF- $\beta$. This metabolic modulation of T cells within the TME results in an impaired anti-tumoural response. IDO, indoleamine 2,3-dioxygenase; OXPHOS, oxidative phosphorylation; PD-1, programmed cell death-1; PD-L1, programmed cell death-ligand 1; TCA cycle, tricarboxylic acid cycle; TGF- $\beta$, transforming growth factor-beta; and TME, tumour microenvironment.

Elevated aerobic glycolysis in tumour cells can have a detrimental impact on intratumoural T cell function (Figure 1). Glucose restriction and the limitation of the glycolytic metabolite phosphoenolpyruvate impairs/reduces calcium and the nuclear factor of activated T cells (NFAT) signalling, impairing the $T$ cell function [17]. Studies overexpressing phosphoenolpyruvate carboxykinase 1 in T cells boosted calcium and NFAT signalling leading to restricted tumour growth in a murine melanoma model [17].

Aside from aerobic glycolysis, the expression of amino acid-metabolising enzymes by tumour cells and tumour-associated cells, deprives local $\mathrm{T}$ cell populations within the TME. In particular, arginine levels within the TME are reduced by tumour cells and tumour-associated macrophages (TAMs) expressing arginine metabolising enzymes such as inducible nitric oxide synthase (iNOS) and arginase, respectively. Moreover, expression by both cell types of indoleamine 2,3-dioxygenase (IDO) depletes tryptophan via the production of kynurenine, thereby further suppressing the T cell function within the TME [24] and promoting the formation of Tregs [6] (Figure 1). Glutamine is another important amino acid required for cancer cell proliferation, which is incorporated into the TCA cycle via anaplerosis where it can contribute to the generation of biosynthetic intermediates such as nucleotides, non-essential amino acids, and lipids [25]. The reliance on glutamine by multiple cancer types makes it an intriguing target therapeutically and glutamine blockade has been reported to be tolerated by effector $\mathrm{T}$ cells, enhancing oxidative metabolism 
and mitochondrial protein expression whilst proving to be detrimental for cancer cells in vivo [26].

More recently, studies have revealed that $\mathrm{T}$ cells within the TME experience persistent antigen exposure under hypoxic conditions leading to detrimental mitochondrial health [27-29]. Mechanistically, the double-edged sword of hypoxia and persistent antigen exposure impairs ADP-coupled oxidative phosphorylation, increases mitochondrial ROS production resulting in an impaired mitochondrial function leading to $\mathrm{T}$ cell exhaustion $[28,29]$. Interestingly, circumvention of this exhausted phenotype can be achieved using anti-oxidants enhancing the anti-tumour activity of chronically stimulated T cells [29].

The combination of nutrient deprivation, hypoxia, persistent antigen exposure, and immunosuppression within the TME has detrimental consequences on the mitochondrial fitness of anti-tumour $\mathrm{T}$ cells $[16,28,30]$ (Figure 1). A further layer of complexity is the engagement of immune checkpoint proteins that can intrinsically modulate $\mathrm{T}$ cell metabolic reprogramming favouring a pro-tumour outcome [31]. For example, ligation of immune checkpoint protein programmed cell death-1 (PD-1) results in altered mitochondrial cristae morphology leading to an upregulation of fatty acid oxidation and subsequently, dysfunction in CD8+ T cells [31-33]. Interestingly, tumours that escape anti-PD1 blockade therapy are able to do so via the suppression of the mitochondrial function in $\mathrm{T}$ cells [34]. Furthermore, tumour-derived TGF- $\beta$ has been shown to negatively impact on the mitochondrial function (via phosphorylation of Smad proteins), resulting in compromised IFN $\gamma$ production and the reduced anti-tumour activity of T cells [16,35] (Figure 1). Collectively, the metabolic modulation of the $\mathrm{T}$ cell mitochondrial function appears critical to immune system evasion and cancer progression.

Therefore, for the future success of CAR-T cell therapy, particularly in a solid tumour setting it is imperative that the metabolic implications of both the tumour and the CAR-T are considered.

\section{Current Metabolic Conditioning of CAR-T Cells}

\subsection{CAR-T Cell Design}

The extracellular domain of a CAR is composed of the single-chain variable fragment of an antibody targeting an antigen expressed on the tumour cell surface, and this is combined with an intracellular T cell receptor (TCR) signalling domain. In first-generation CARs, this signalling domain is conventionally composed of the $\mathrm{CD} 3 \zeta$ chain of the TCR alone, whereas successive second or third generations of CARs include one or more costimulatory domains, such as CD28 or 4-1BB [36] (Figure 2). CAR design itself can have an impact on CAR-T cell function, with the choice of a co-stimulatory domain governing CAR-T cell metabolism. CAR-T cells with a CD28 co-stimulatory domain (Yescarta) show enhanced aerobic glycolysis and effector memory differentiation [37]. Whereas the inclusion of a 4-1BB co-stimulatory domain rather than CD28 (Kymriah, UCART19, Lisocel) promotes an oxidative metabolism and increases mitochondrial biogenesis [37]. This metabolic reprogramming results in enhanced central memory differentiation and improves CAR-T cell proliferation and persistence in vitro. The importance of co-stimulatory domain selection is evidenced by the differential downstream metabolic programs they govern. For instance, one particular co-stimulatory domain may be more beneficial at treating a certain malignancy with a particular metabolic program. Furthermore, consideration should be given towards the quality of downstream signal transduction (low or high affinity binding) and its influence on downstream CAR-T cell metabolism. Identifying differences in signal transduction and subsequent metabolic reprogramming may give insight into CAR-T cell functional differences and exhaustion profiles with therapeutic value. 


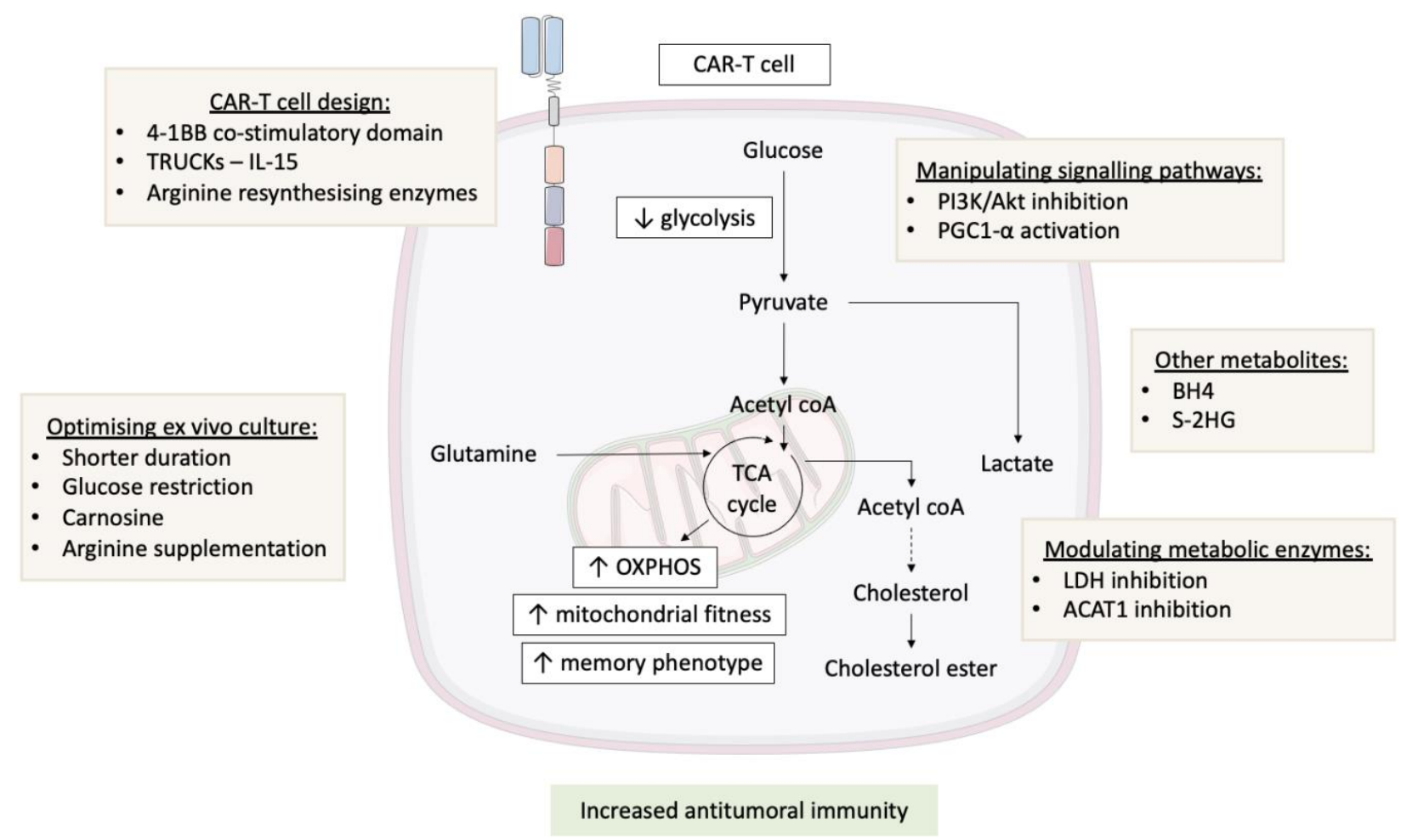

Figure 2. Metabolic optimisation of CAR-T cells. Various strategies are currently being investigated to enhance the metabolic program of CAR-T cells and overcome the metabolic hostility of the TME. This includes improving CAR-T cell design, optimising ex vivo culture conditions, manipulating signalling pathways, and modulating various metabolic enzymes. These strategies increase OXPHOS levels and mitochondrial fitness in CAR-T cells leading to an enhanced central memory $\mathrm{T}$ cell compartment, resulting in a fitter CAR-T cell product with improved anti-tumoural immunity. ACAT1, cholesterol acyltransferase 1; $\mathrm{BH} 4$, tetrahydrobiopterin; CAR-T cell, chimeric antigen receptor T cell; $\mathrm{LDH}$, lactate dehydrogenase; OXPHOS, oxidative phosphorylation; PGC1- $\alpha$, PPAR-gamma coactivator 1- $\alpha$; S-2HG, S-2-hydroxygluterate; TCA cycle, tricarboxylic acid cycle; TME, tumour microenvironment; and TRUCKs, T-cells redirected for unrestricted cytokine-mediated killing.

Next generation (fourth generation) CAR-T cells are known as T-cell redirected for unrestricted cytokine-mediated killing (TRUCKs) and incorporate a transgenic cytokine release system designed to improve proliferation and persistence in vivo [38] (Figure 2). Typically, the cytokines incorporated include IL-12, IL-15, and IL-18, most of which also have known metabolic effects on T cells. For example, CAR-T cells expanded in the presence of IL-15 have decreased mTORC1 activity, which results in a reduction in glycolysis. IL15-treated CAR-T cells also displayed elevated levels of OXPHOS and enhanced spare respiratory capacity, as well as an increased expression of fatty acid oxidation-related genes. These metabolic changes result in less differentiated CAR-T cells that maintain a stem cell memory phenotype and enhance CAR-T cell proliferation, improving their in vivo anticancer activity and longevity [39].

Whilst a specific CAR-T cell design has clear implications on the metabolic program of the product, another important consideration is how these CAR-T cells are subsequently expanded and maintained prior to patient infusion.

\subsection{Optimising Media}

One of the major factors limiting the efficacy of CAR-T cell therapy is the metabolic hostility of the TME. CAR-T cells face the same metabolic challenges and immunosuppression within the TME as host $\mathrm{T}$ cells, having to compete with tumour cells for nutrients and overcome suppression by various metabolites. In addition, the suppressive TME may initially impact on the homing capacity of CAR-T cells. Enhancing CAR-T cell memory is key 
to improving their persistence and anti-tumour activity and increasing their therapeutic capacity. CAR-T cells are currently created, expanded, and maintained in media formulation containing excessive nutrient levels such as carbohydrates and amino acids [4,9]. In particular, two supraphysiological nutrients are glucose and glutamine, which are essential fuels for rapidly proliferating, activated immune cells [13,40]. Clinical and experimental media formulations do not (i) recapitulate the nutrient hostile, immunosuppressive environment of the TME, including any abundant oncometabolites or (ii) rescue any $\mathrm{T}$ cells with long-lasting metabolic defects inflicted from pre-cancer treatments such as chemotherapy and radiotherapy.

Efforts have been made to move away from the reliance on human serum in media used for CAR-T cell manufacturing with the identification of a concentrated growth factor derived from human transfusion grade whole blood fractions, known as PhysiologixTM xeno-free (XF) hGFC (Phx) [9]. Phx, in comparison to human serum, has been demonstrated to enrich carnosine levels that enhance downstream $\mathrm{T}$ cell proliferation and peripheral blood abundance in both clinical and research grade media. Furthermore, the authors demonstrate that carnosine inclusion in media formulations are able to neutralise extracellular protons $\left(\mathrm{H}^{+}\right)$derived from lactate (via aerobic glycolysis), promoting a metabolic transition from glycolysis towards an oxidative phenotype in CAR-T cells, thus supporting its inclusion in media formulation [9]. Interestingly, previous methods that curtail T cell glycolysis levels ex vivo promote anti-tumour activity, highlighting the potential importance of including carnosine in CAR-T cell clinical culture methods [41]. A summary of optimising ex vivo culture can be found in Figure 2.

Moreover, shortening the duration of CAR-T cell expansion ex vivo has also been shown to limit their differentiation resulting in CAR-T cells with an enhanced proliferation and effector function in vitro. Importantly, these CAR-T cells also displayed enhanced persistence and improved antileukemic activity in a mouse acute lymphoblastic leukaemia (ALL) xenograft model [42]. It is clear that in vitro expansion and culture of CAR-T cells considerably impacts on their phenotype and function. To counteract this, efforts are being made to generate in vivo CAR-T cells, thus removing the non-physiological culture conditions in vitro CAR-T cells are exposed to [43]. For instance, lentiviral vectors can be delivered specifically to CD8+ T cells in vivo, generating CD19-CAR-T cells with effective B-cell elimination [44].

\subsection{Lactate}

Lactate is one of the most abundant metabolites within the solid TME with levels reaching up to a staggering $40 \mathrm{mM}$ [45]. As discussed previously, lactate has detrimental effects on the immune system, that ultimately reduces the anti-tumour response, specifically promoting the differentiation of Tregs [22]. Whether contributions of lactate within the TME can promote the formation of a CAR-Treg phenotype, compromising the anti-tumour response is yet to be determined.

The recently-developed method of in vivo metabolomics by Ma et al., could potentially identify the contribution of lactate-derived carbons towards central metabolic pathways in CAR-T cells [46]. Given the reported differences of T cell metabolism between in vitro and in vivo, the technique of in vivo metabolomics could be used to inform downstream methods of optimising CAR-T cell metabolism for therapeutic success [46].

Whilst little is known about the direct influence of lactate on CAR-T cell metabolism and function, in an attempt to overcome the suppressive properties of lactate, studies have begun to investigate the mechanistic effect of lactate dehydrogenase (LDH) inhibition. Specifically, the inhibition of LDH enhances the CAR-T cell function $[47,48]$, in particular one study identified a synergistic effect with IL-21 to promote CAR-T memory and enhanced downstream anti-tumour immunity [48]. 


\subsection{Arginine}

CAR-T cell metabolism may also be manipulated to overcome low arginine levels within the TME.

Supplementing the media with L-arginine during ex vivo culture also has the potential to improve CAR-T cell therapy [49] via increased intracellular levels, driving a shift in CAR-T cell metabolism from glycolysis towards OXPHOS. This metabolic shift promotes the formation of central memory-like $\mathrm{T}$ cells, with enhanced survival and improved antitumour activity in vivo. Another method developed to overcome limited in vivo arginine levels is the engineering of CAR-T cells to express the arginine resynthesizing enzymes argininosuccinate synthase (ASS) and ornithine transcarbamylase (OTC). These modifications alongside the CAR itself can enhance the proliferation and improved anti-tumour activity in mouse models [24].

\subsection{Glucose Restriction}

To date, most studies have reported an association between elevated OXPHOS and the enhanced formation of the central memory CAR-T cell compartment leading to improved treatment outcomes [50,51]. Therefore, methods to boost CAR-T cell OXPHOS levels both ex vivo and in vivo have been under scrutiny [52,53]. Interestingly, glucose restriction via the substitution of glucose for another carbohydrate such as galactose in cell culture media has previously been demonstrated to boost OXPHOS levels in T cells and NK cells $[40,54]$. Furthermore, a recent study has investigated a period of glucose restriction after activation during CAR-T cell expansion as a potential strategy to enhance the efficacy of CAR-T cell therapy [55]. Antigen-specific CD8+ effector T cells subjected to transient glucose restriction (TGR) displayed enhanced tumour clearance in a mouse model of lymphoma, with an increase in the number of donor CD8+ $\mathrm{T}$ cell in circulation that persisted up to 20 days after adoptive transfer. Additionally, CAR-T cells subjected to TGR displayed an increased production of the effector molecules IFN $\gamma$ and granzyme B [55]. This enhanced persistence and anti-tumour activity is underpinned by metabolic rewiring, with TGR CD8+ effector T cells undergoing a metabolic shift towards OXPHOS and showing an enhanced anabolic phenotype upon re-exposure to glucose.

A further consequence of glucose restricted $\mathrm{T}$ cells is the ability to utilise inosine [56]. T cells exhibit metabolic plasticity during glucose restriction, incorporating inosine-metabolised carbons into central metabolic pathways to generate adenosine triphosphate and biosynthetic intermediates resulting in an enhanced oxidative metabolism [56].

Whether TGR is clinically relevant is yet to be determined, however there are multiple considerations as to how this could be achieved. Given that glucose is essential to generate clinically sufficient numbers of CAR-T cells for infusion, it is unlikely that an early period of TGR would be a success. However, whether the introduction of TGR (for example with a galactose substitution) prior to infusion would generate a therapeutic avenue is an interesting thought. Furthermore, whether this method could be further refined by utilising a mixed culture system, with half the CAR-T cells cultured under standard clinical conditions and half under TGR to generate a fitter product remains to be seen.

\subsection{Other Metabolites}

It is likely that multiple metabolites could influence the generation of a fitter CAR-T cell product in the short- and long-term. Glutamine is a critical, non-essential amino acid required for $\mathrm{T}$ cell function [13], and whilst it is thought that amino acid competition exists between the tumour and T cells [5], little is known about the role of glutamine in CAR-T cell culture and function. A study recently described a novel glutamine antagonist, JHU083, that suppresses both glycolytic and oxidative metabolic pathways in tumour cells, leading to a reduction in hypoxia, acidosis, and nutrient depletion [26]. Surprisingly, glutamine blockade using JHU0833 promoted elevated effector T cell oxidative metabolism and longevity within the TME [26]. This important study highlights that intratumoural $\mathrm{T}$ cells are able to demonstrate metabolic plasticity with regards to glutamine 
metabolism becoming dispensable and would therefore certainly be an interesting avenue to pursue in combination with CAR-T cells [26]. Furthermore, T cells cultured ex vivo in glutamine-depleted media demonstrated robust anti-tumour activity upon adoptive transfer in mice. These studies highlight the requirement for investigation into the role of glutamine manipulation in CAR-T cell culture conditions pre-infusion [57].

The S-enantiomer of 2-hydroxygluterate (S-2HG) is an immunometabolite that is produced following HIF- $1 \alpha$ stabilisation. S-2HG has a role in regulating T cell fate, inhibiting their differentiation into effector cells. Expanding CAR-T cells in the presence of S-2HG yields increased proportions of central memory cells, regardless of the co-stimulatory domain included in the CAR design. Importantly, the in vitro cytotoxic activity and cytokine production of S-2HG treated CAR-T cells was not impaired, and in a mouse xenograft model, CAR-T cells treated with S-2HG display enhanced anti-tumour activity and reduced tumour progression [58].

Another beneficial metabolite identified, tetrahydrobiopterin (BH4), can be produced by activated $\mathrm{T}$ cells and has a role in the modulation of iron metabolism and mitochondrial bioenergetics [59]. Intriguingly, kynurenine-mediated $\mathrm{T}$ cell immunosuppression can be rescued by $\mathrm{BH} 4$, outlining its importance in anti-tumour immunity and raising further questions about its inclusion in CAR-T cell design [59].

Whilst the primary focus of most studies revolves around the interplay of glucose and amino acid metabolism between the tumour and the CAR-T cells, another important biomolecule to consider is cholesterol. Cholesterol is an integral component of the cell membrane, essential for $\mathrm{T}$ cell proliferation [60]. The inhibition of cholesterol esterification by the enzyme cholesterol acyltransferase 1 (ACAT1) utilising the repurposed drug avasimibe leads to an improved anti-tumour response by CD8+ T cells that further enhanced outcomes in combination therapy with anti-PD-1 treatment [61]. Such findings have now been translated into the CAR-T cell field, whereby siRNA targeting of ACAT1 in a mesothelin-expressing pancreatic carcinoma model reduced tumour volume, elevated IFN $\gamma$ levels, and improved overall cancer targeting in vivo [62]. Repurposing avasimibe (atherosclerosis treatment, clinically) as a combination therapy with CAR-T cells is an attractive avenue given the known safety profiles in humans [61].

\subsection{Manipulating Signalling Pathways}

Successive generations of CAR-T cells have been designed to incorporate downstream signalling enhancement. One pathway of interest, the PI3K/Akt signalling pathway, has previously been implicated in upregulating glycolysis levels in stimulated human $\mathrm{T}$ cell subsets $[13,14]$. In a CD33 targeted CAR-T cell model of acute myeloid leukaemia, the authors identified upregulation of PI3K/Akt/mTOR and glycolytic gene expression in ex vivo expanded $\mathrm{T}$ cells in comparison to control $\mathrm{T}$ cells.

The PI3K/Akt/mTOR pathway is associated with a terminal effector T cell differentiation that can hinder CAR-T cell success in comparison to the central memory phenotype [63] (Figure 2). As decreasing T cell glycolysis levels ex vivo is associated with a fitter T cell product in vivo, in an attempt to circumvent this, the authors utilised a panel of inhibitors targeting PI3K, Akt, mTOR, and the glycolysis pathway [50]. Here, the authors observed increased naïve and central memory $\mathrm{T}$ cell numbers with largely decreased terminal effector cells. Whilst the inhibitors employed had a positive effect on the desired $\mathrm{T}$ cell compartments, only PI3K inhibition maintained cell numbers in comparison to the control. In corroboration with the PI3K inhibitor, another study investigated the role of Akt inhibition in depth. Here, the authors elegantly show that Akt inhibition elevates FOXO1 expression resulting in heightened CD62L-expressing central memory $\mathrm{T}$ cells. These findings were accompanied with a reduced glycolytic gene signature and lowered glycolysis levels in a human's anti-CD19 CAR-T cells [64].

Small-molecule inhibitors of PI3K have failed to enter the clinic for a range of cancers due to limited stability, poor specificity, and high toxicity, and are therefore unlikely to 
compliment CAR-T cell therapy in vivo, however it could be an ex vivo avenue for CAR-T cell optimization $[65,66]$.

Akt along with other signalling molecules such as AMP-activated protein kinase (AMPK), p38 MAPK, and SIRT1 can post-transcriptionally modify the PPAR-gamma coactivator 1- $\alpha$ (PGC1- $\alpha)$ [16]. These modifications can increase mitochondrial biogenesis and OXPHOS levels. Furthermore, T cells with an enforced expression of PGC1- $\alpha$, improve mitochondrial biogenesis, cytokine secretion, and intratumoural $\mathrm{T}$ cell function in vivo, counteracting the impaired mitochondrial OXPHOS observed in dysfunctional intratumoural T cells [16]. Potential activation of PGC1- $\alpha$ is already implemented in CAR-T cell design, as an engagement of the co-stimulatory molecule, 4-1BB has been demonstrated to enhance PGC1- $\alpha$ levels dependent on p38-MAPK in T cells [67]. Methods that can further enhance levels of PGC1- $\alpha$ activation certainly warrant investigation in CAR-T cell design (Figure 2).

\section{Concluding Remarks}

It is known that the TME can shape the metabolic and phenotypic fitness of CAR$\mathrm{T}$ cells. This hostile, immunosuppressive microenvironment leads to an unfavourable response when utilising CAR-T cells. Therefore, investigating the metabolic and phenotypic fitness of CAR-T cells in response to the TME is essential in order to improve the efficacy of CAR-T cells. It is now clear that there are many ways that CAR-T cell metabolism can be manipulated for therapeutic gain. Multiple in vitro studies have reported that boosting oxidative metabolism and mitochondrial fitness leads to an increased central memory $\mathrm{T}$ cell compartment resulting in a fitter CAR-T cell product and improved outcomes, however many of these strategies are yet to be tested in clinical trials. Furthermore, questions still remain about the impact of the CAR-T cell manufacturing process ultimately on the end product's metabolism, for example expansion in the presence of various cytokines such as IL-2, IL-7, or IL-15, the use of bioreactors vs. bags, the type of stimulus utilised to activate the modified T cells, implications of using peripheral blood mononuclear cells or individually isolated $\mathrm{T}$ cell populations, and whether using antigen specific $\mathrm{T}$ cells stifles any metabolic heterogeneity.

Current CAR-T cell design has incorporated the three traditional signals of $\mathrm{T}$ cell activation (signal 1; TCR, signal 2; co-stimulatory, and signal 3; cytokine stimulation) with somewhat unintentional effects on CAR-T cell metabolism. However, whether future generations of clinical CAR-T cell design could include a metabolically orientated 'fourth signal', such as overexpression of particular nutrient transporters or metabolic enzymes with the hope of generating a fitter product, is of particular interest. An additional factor to consider would be CAR-T cell therapy in combination with a metabolic modulator to potentially further improve efficacy. Whilst there are a multitude of metabolic modulators to pursue, considerable attention is required to demonstrate no detrimental effects on the CAR-T cells compromising function. We envisage the identification of therapeutic windows that could be developed to circumvent any interfering effects.

The future of next generation CAR-T cell therapies is an exciting area for the treatment of malignancy. Given such promising results in haematological cancers such as B cell lymphomas [68], there is much to look ahead to with regards to the treatment of solid tumours. The re-emergence of the fields of immunometabolism and oncometabolism over the last 10-15 years provide us with a platform to further enhance CAR-T cell therapy and improve clinical outcomes in the fight against cancer.

Author Contributions: Conceptualization, N.J.; Writing-Original Draft Preparation, Y.J., N.J.; Writing-Review \& Editing, Y.J., J.Z., O.O., N.J.; Supervision, N.J. All authors have read and agreed to the published version of the manuscript.

Funding: This research received no external funding.

Institutional Review Board Statement: Not applicable. 
Informed Consent Statement: Not applicable.

Data Availability Statement: Not applicable.

Conflicts of Interest: The authors declare no conflict of interest.

\section{References}

1. June, C.H.; O'Connor, R.S.; Kawalekar, O.U.; Ghassemi, S.; Milone, M.C. CAR T cell immunotherapy for human cancer. Science 2018, 359, 1361-1365. [CrossRef]

2. Boyiadzis, M.M.; Dhodapkar, M.V.; Brentjens, R.J.; Kochenderfer, J.N.; Neelapu, S.S.; Maus, M.V.; Porter, D.L.; Maloney, D.G.; Grupp, S.A.; Mackall, C.L.; et al. Chimeric antigen receptor (CAR) T therapies for the treatment of hematologic malignancies: Clinical perspective and significance. J. Immunother. Cancer 2018, 6, 137. [CrossRef] [PubMed]

3. Depil, S.; Duchateau, P.; Grupp, S.A.; Mufti, G.; Poirot, L. 'Off-the-shelf' allogeneic CAR T cells: Development and challenges. Nat. Rev. Drug Discov. 2020, 19, 185-199. [CrossRef] [PubMed]

4. Xu, Q.; Harto, H.; Berahovich, R.; Xu, S.; Zhou, H.; Golubovskaya, V.; Wu, L. Generation of CAR-T Cells for Cancer Immunotherapy. Adv. Struct. Saf. Stud. 2018, 1884, 349-360. [CrossRef]

5. Chang, C.-H.; Qiu, J.; O'Sullivan, D.; Buck, M.D.; Noguchi, T.; Curtis, J.D.; Chen, Q.; Gindin, M.; Gubin, M.M.; Van Der Windt, G.J.; et al. Metabolic Competition in the Tumor Microenvironment Is a Driver of Cancer Progression. Cell 2015, 162, 1229-1241. [CrossRef] [PubMed]

6. Routy, J.-P.; Routy, B.; Graziani, G.M.; Mehraj, V. The Kynurenine Pathway is a Double-Edged Sword in Immune-Privileged Sites and in Cancer: Implications for Immunotherapy. Int. J. Tryptophan Res. 2016, 9, 67-77. [CrossRef] [PubMed]

7. Kedia-Mehta, N.; Finlay, D.K. Competition for nutrients and its role in controlling immune responses. Nat. Commun. 2019, 10, 1-8. [CrossRef] [PubMed]

8. Das, R.K.; O'Connor, R.S.; Grupp, S.A.; Barrett, D.M. Lingering effects of chemotherapy on mature T cells impair proliferation. Blood Adv. 2020, 4, 4653-4664. [CrossRef]

9. Ghassemi, S.; Martinez-Becerra, F.J.; Master, A.M.; Richman, S.A.; Heo, D.; Leferovich, J.; Tu, Y.; García-Cañaveras, J.C.; Ayari, A.; Lu, Y.; et al. Enhancing Chimeric Antigen Receptor T Cell Anti-tumor Function through Advanced Media Design. Mol. Ther. Methods Clin. Dev. 2020, 18, 595-606. [CrossRef]

10. Wagner, J.; Wickman, E.; DeRenzo, C.; Gottschalk, S. CAR T Cell Therapy for Solid Tumors: Bright Future or Dark Reality? Mol. Ther. 2020, 28, 2320-2339. [CrossRef]

11. O'Neill, L.A.J.; Kishton, R.J.; Rathmell, R.J.K.J. A guide to immunometabolism for immunologists. Nat. Rev. Immunol. 2016, 16, 553-565. [CrossRef]

12. Buck, M.D.; O'Sullivan, D.; Pearce, E.L. T cell metabolism drives immunity. J. Exp. Med. 2015, 212, 1345-1360. [CrossRef]

13. Jones, N.; Vincent, E.E.; Cronin, J.G.; Panetti, S.; Chambers, M.; Holm, S.R.; Owens, S.E.; Francis, N.J.; Finlay, D.K.; Thornton, C.A. Akt and STAT5 mediate naïve human CD4+ T-cell early metabolic response to TCR stimulation. Nat. Commun. 2019, 10, 1-13. [CrossRef]

14. Gubser, P.M.; Bantug, G.R.; Razik, L.; Fischer, M.; Dimeloe, S.; Hoenger, G.; Durovic, B.; Jauch, A.; Hess, C. Rapid effector function of memory CD8+ T cells requires an immediate-early glycolytic switch. Nat. Immunol. 2013, 14, 1064-1072. [CrossRef]

15. Balmer, M.L.; Hess, C. Starving for survival-How catabolic metabolism fuels immune function. Curr. Opin. Immunol. 2017, 46, 8-13. [CrossRef]

16. Scharping, N.E.; Menk, A.V.; Moreci, R.S.; Whetstone, R.D.; Dadey, R.E.; Watkins, S.C.; Ferris, R.L.; Delgoffe, G.M. The Tumor Microenvironment Represses T Cell Mitochondrial Biogenesis to Drive Intratumoral T Cell Metabolic Insufficiency and Dysfunction. Immunity 2016, 45, 374-388. [CrossRef]

17. Ho, P.-C.; Bihuniak, J.D.; MacIntyre, A.N.; Staron, M.; Liu, X.; Amezquita, R.; Tsui, Y.-C.; Cui, G.; Micevic, G.; Perales, J.C.; et al. Phosphoenolpyruvate Is a Metabolic Checkpoint of Anti-tumor T Cell Responses. Cell 2015, 162, 1217-1228. [CrossRef]

18. Harmon, C.; Robinson, M.W.; Hand, F.; AlMuaili, D.; Mentor, K.; Houlihan, D.D.; Hoti, E.; Lynch, L.; Geoghegan, J.; O’Farrelly, C. Lactate-Mediated Acidification of Tumor Microenvironment Induces Apoptosis of Liver-Resident NK Cells in Colorectal Liver Metastasis. Cancer Immunol. Res. 2018, 7, 335-346. [CrossRef]

19. Wu, H.; Estrella, V.; Beatty, M.; Abrahams, D.; El-Kenawi, A.; Russell, S.; Ibrahim-Hashim, A.; Longo, D.L.; Reshetnyak, Y.K.; Moshnikova, A.; et al. T-cells produce acidic niches in lymph nodes to suppress their own effector functions. Nat. Commun. 2020, 11, 1-13. [CrossRef]

20. Sugiura, A.; Rathmell, J.C. Metabolic Barriers to T Cell Function in Tumors. J. Immunol. 2018, 200, 400-407. [CrossRef]

21. Quinn, W.J.; Jiao, J.; TeSlaa, T.; Stadanlick, J.; Wang, Z.; Wang, L.; Akimova, T.; Angelin, A.; Schäfer, P.M.; Cully, M.D.; et al. Lactate Limits T Cell Proliferation via the NAD $(\mathrm{H})$ Redox State. Cell Rep. 2020, 33, 108500. [CrossRef] [PubMed]

22. Angelin, A.; Gil-De-Gómez, L.; Dahiya, S.; Jiao, J.; Guo, L.; Levine, M.H.; Wang, Z.; Quinn, W.J.; Kopinski, P.K.; Wang, L.; et al. Foxp3 Reprograms T Cell Metabolism to Function in Low-Glucose, High-Lactate Environments. Cell Metab. 2017, 25, 1282-1293.e7. [CrossRef] [PubMed]

23. Renner, K.; Bruss, C.; Schnell, A.; Koehl, G.; Becker, H.M.; Fante, M.; Menevse, A.-N.; Kauer, N.; Blazquez, R.; Hacker, L.; et al. Restricting Glycolysis Preserves T Cell Effector Functions and Augments Checkpoint Therapy. Cell Rep. 2019, 29, 135-150.e9. [CrossRef] [PubMed] 
24. Fultang, L.; Booth, S.; Yogev, O.; da Costa, B.M.; Tubb, V.; Panetti, S.; Stavrou, V.; Scarpa, U.; Jankevics, A.; Lloyd, G.; et al. Metabolic engineering against the arginine microenvironment enhances CAR-T cell proliferation and therapeutic activity. Blood 2020, 136, 1155-1160. [CrossRef]

25. Jiang, J.; Srivastava, S.; Zhang, J. Starve Cancer Cells of Glutamine: Break the Spell or Make a Hungry Monster? Cancers 2019, 11, 804. [CrossRef]

26. Leone, R.D.; Zhao, L.; Englert, J.M.; Sun, I.-M.; Oh, M.-H.; Arwood, M.L.; Bettencourt, I.A.; Patel, C.H.; Wen, J.; Tam, A.; et al. Glutamine blockade induces divergent metabolic programs to overcome tumor immune evasion. Science 2019, 366, 1013-1021. [CrossRef]

27. Yu, Y.-R.; Imrichova, H.; Wang, H.; Chao, T.; Xiao, Z.; Gao, M.; Rincon-Restrepo, M.; Franco, F.; Genolet, R.; Cheng, W.-C.; et al. Disturbed mitochondrial dynamics in CD8+ TILs reinforce T cell exhaustion. Nat. Immunol. 2020, 21, 1540-1551. [CrossRef]

28. Vardhana, S.A.; Hwee, M.A.; Berisa, M.; Wells, D.K.; Yost, K.E.; King, B.; Smith, M.; Herrera, P.S.; Chang, H.Y.; Satpathy, A.T.; et al. Impaired mitochondrial oxidative phosphorylation limits the self-renewal of T cells exposed to persistent antigen. Nat. Immunol. 2020, 21, 1022-1033. [CrossRef]

29. Scharping, N.E.; Rivadeneira, D.B.; Menk, A.V.; Vignali, P.D.A.; Ford, B.R.; Rittenhouse, N.L.; Peralta, R.; Wang, Y.; Wang, Y.; Depeaux, K.; et al. Mitochondrial stress induced by continuous stimulation under hypoxia rapidly drives $\mathrm{T}$ cell exhaustion. Nat. Immunol. 2021, 22, 205-215. [CrossRef]

30. Liu, Y.-N.; Yang, J.-F.; Huang, D.-J.; Ni, H.-H.; Zhang, C.-X.; Zhang, L.; He, J.; Gu, J.-M.; Chen, H.-X.; Mai, H.-Q.; et al. Hypoxia Induces Mitochondrial Defect That Promotes T Cell Exhaustion in Tumor Microenvironment Through MYC-Regulated Pathways. Front. Immunol. 2020, 11. [CrossRef]

31. Patsoukis, N.; Bardhan, K.; Chatterjee, P.; Sari, D.; Liu, B.; Bell, L.N.; Karoly, E.D.; Freeman, G.J.; Petkova, V.; Seth, P.; et al. PD-1 alters T-cell metabolic reprogramming by inhibiting glycolysis and promoting lipolysis and fatty acid oxidation. Nat. Commun. 2015, 6, 6692. [CrossRef]

32. Ogando, J.; Sáez, M.E.; Santos, J.; Nuevo-Tapioles, C.; Gut, M.; Esteve-Codina, A.; Heath, S.; González-Pérez, A.; Cuezva, J.M.; LaCalle, R.A.; et al. PD-1 signaling affects cristae morphology and leads to mitochondrial dysfunction in human CD8+ T lymphocytes. J. Immunother. Cancer 2019, 7, 151. [CrossRef]

33. Thommen, D.S.; Koelzer, V.H.; Herzig, P.; Roller, A.; Trefny, M.; Dimeloe, S.; Kiialainen, A.; Hanhart, J.; Schill, C.; Hess, C.; et al. A transcriptionally and functionally distinct PD-1+CD8+ T cell pool with predictive potential in non-small-cell lung cancer treated with PD-1 blockade. Nat. Med. 2018, 24, 994-1004. [CrossRef]

34. Kumar, A.; Chamoto, K.; Chowdhury, P.S.; Honjo, T. Tumors attenuating the mitochondrial activity in T cells escape from PD-1 blockade therapy. eLife 2020, 9, e52330. [CrossRef]

35. Dimeloe, S.; Gubser, P.; Loeliger, J.; Frick, C.; Develioglu, L.; Fischer, M.; Marquardsen, F.; Bantug, G.R.; Thommen, D.; Lecoultre, Y.; et al. Tumor-derived TGF- $\beta$ inhibits mitochondrial respiration to suppress IFN- $\gamma$ production by human CD4+ T cells. Sci. Signal. 2019, 12, eaav3334. [CrossRef]

36. Irving, M.; De Silly, R.V.; Scholten, K.; Dilek, N.; Coukos, G. Engineering Chimeric Antigen Receptor T-Cells for Racing in Solid Tumors: Don't Forget the Fuel. Front. Immunol. 2017, 8, 267. [CrossRef]

37. Kawalekar, O.U.; O'Connor, R.S.; Fraietta, J.A.; Guo, L.; Mcgettigan, S.E.; Posey, A.D.; Patel, P.R.; Guedan, S.; Scholler, J.; Keith, B.; et al. Distinct Signaling of Coreceptors Regulates Specific Metabolism Pathways and Impacts Memory Development in CAR T Cells. Immunity 2016, 44, 380-390. [CrossRef]

38. Petersen, C.T.; Krenciute, G. Next Generation CAR T Cells for the Immunotherapy of High-Grade Glioma. Front. Oncol. 2019, 9 , 69. [CrossRef]

39. Alizadeh, D.; Wong, R.A.; Yang, X.; Wang, D.; Pecoraro, J.R.; Kuo, C.-F.; Aguilar, B.; Qi, Y.; Ann, D.K.; Starr, R.; et al. IL15 Enhances CAR-T Cell Antitumor Activity by Reducing mTORC1 Activity and Preserving Their Stem Cell Memory Phenotype. Cancer Immunol. Res. 2019, 7, 759-772. [CrossRef]

40. Chang, C.-H.; Curtis, J.D.; Maggi, L.B.; Faubert, B.; Villarino, A.V.; O’Sullivan, D.; Huang, S.C.-C.; Van Der Windt, G.J.; Blagih, J.; Qiu, J.; et al. Posttranscriptional Control of T Cell Effector Function by Aerobic Glycolysis. Cell 2013, 153, 1239-1251. [CrossRef]

41. Sukumar, M.; Liu, J.; Ji, Y.; Subramanian, M.; Crompton, J.G.; Yu, Z.; Roychoudhuri, R.; Palmer, D.C.; Muranski, P.; Karoly, E.D.; et al. Inhibiting glycolytic metabolism enhances CD8+ T cell memory and antitumor function. J. Clin. Investig. 2013, 123, 4479-4488. [CrossRef]

42. Ghassemi, S.; Nunez-Cruz, S.; O'Connor, R.S.; Fraietta, J.A.; Patel, P.R.; Scholler, J.; Barrett, D.M.; Lundh, S.M.; Davis, M.M.; Bedoya, F.; et al. Reducing Ex Vivo Culture Improves the Antileukemic Activity of Chimeric Antigen Receptor (CAR) T Cells. Cancer Immunol. Res. 2018, 6, 1100-1109. [CrossRef]

43. Frank, A.M.; Braun, A.H.; Scheib, L.; Agarwal, S.; Schneider, I.C.; Fusil, F.; Perian, S.; Sahin, U.; Thalheimer, F.B.; Verhoeyen, E.; et al. Combining T-cell-specific activation and in vivo gene delivery through CD3-targeted lentiviral vectors. Blood Adv. 2020, 4, 5702-5715.

44. Pfeiffer, A.; Thalheimer, F.B.; Hartmann, S.; Frank, A.M.; Bender, R.R.; Danisch, S.; Costa, C.; Wels, W.S.; Modlich, U.; Stripecke, R.; et al. In vivo generation of human CD 19- CAR T cells results in B-cell depletion and signs of cytokine release syndrome. EMBO Mol. Med. 2018, 10, e9158. [CrossRef]

45. Romero-Garcia, S.; Moreno-Altamirano, M.M.B.; Prado-Garcia, H.; Sánchez-García, F.J. Lactate Contribution to the Tumor Microenvironment: Mechanisms, Effects on Immune Cells and Therapeutic Relevance. Front. Immunol. 2016, 7, 52. [CrossRef] 
46. Ma, E.H.; Verway, M.J.; Johnson, R.M.; Roy, D.G.; Steadman, M.; Hayes, S.; Williams, K.S.; Sheldon, R.D.; Samborska, B.; Kosinski, P.A.; et al. Metabolic Profiling Using Stable Isotope Tracing Reveals Distinct Patterns of Glucose Utilization by Physiologically Activated CD8+ T Cells. Immunity 2019, 51, 856-870.e5. [CrossRef]

47. Mane, M.M.; Cohen, I.J.; Ackerstaff, E.; Shalaby, K.; Ijoma, J.N.; Ko, M.; Maeda, M.; Alberg, A.S.; Vemuri, K.; Satagopan, J.; et al. Lactate Dehydrogenase A Depletion Alters MyC-CaP Tumor Metabolism, Microenvironment, and CAR T Cell Therapy. Mol. Ther. Oncolytics 2020, 18, 382-395. [CrossRef]

48. Hermans, D.; Gautam, S.; García-Cañaveras, J.C.; Gromer, D.; Mitra, S.; Spolski, R.; Li, P.; Christensen, S.; Nguyen, R.; Lin, J.-X.; et al. Lactate dehydrogenase inhibition synergizes with IL-21 to promote CD8+T cell stemness and antitumor immunity. Proc. Natl. Acad. Sci. USA 2020, 117, 6047-6055. [CrossRef]

49. Geiger, R.; Rieckmann, J.C.; Wolf, T.; Basso, C.; Feng, Y.; Fuhrer, T.; Kogadeeva, M.; Picotti, P.; Meissner, F.; Mann, M.; et al. L-Arginine Modulates T Cell Metabolism and Enhances Survival and Anti-tumor Activity. Cell 2016, 167, 829-842.e13. [CrossRef]

50. Zheng, W.; O'Hear, C.E.; Alli, R.; Basham, J.H.; Abdelsamed, H.A.; Palmer, L.E.; Jones, L.L.; Youngblood, B.; Geiger, T.L. PI3K orchestration of the in vivo persistence of chimeric antigen receptor-modified T cells. Leukemia 2018, 32, 1157-1167. [CrossRef]

51. Klebanoff, C.A.; Gattinoni, L.; Torabi-Parizi, P.; Kerstann, K.; Cardones, A.R.; Finkelstein, S.E.; Palmer, D.C.; Antony, P.A.; Hwang, S.T.; Rosenberg, S.A.; et al. Central memory self/tumor-reactive CD8+ T cells confer superior antitumor immunity compared with effector memory T cells. Proc. Natl. Acad. Sci. USA 2005, 102, 9571-9576. [CrossRef] [PubMed]

52. Van Bruggen, J.A.C.; Martens, A.W.J.; Fraietta, J.A.; Hofland, T.; Tonino, S.H.; Eldering, E.; Levin, M.-D.; Siska, P.J.; Endstra, S.; Rathmell, J.C.; et al. Chronic lymphocytic leukemia cells impair mitochondrial fitness in CD8+ T cells and impede CAR T-cell efficacy. Blood 2019, 134, 44-58. [CrossRef] [PubMed]

53. Li, W.; Zhang, L. Rewiring Mitochondrial Metabolism for CD8+ T Cell Memory Formation and Effective Cancer Immunotherapy. Front. Immunol. 2020, 11. [CrossRef] [PubMed]

54. Donnelly, R.P.; Loftus, R.M.; Keating, S.E.; Liou, K.T.; Biron, C.A.; Gardiner, C.M.; Finlay, D.K. mTORC1-Dependent Metabolic Reprogramming Is a Prerequisite for NK Cell Effector Function. J. Immunol. 2014, 193, 4477-4484. [CrossRef]

55. Geltink, R.I.K.; Edwards-Hicks, J.; Apostolova, P.; O'Sullivan, D.; Sanin, D.E.; Patterson, A.E.; Puleston, D.J.; Ligthart, N.A.M.; Buescher, J.M.; Grzes, K.M.; et al. Metabolic conditioning of CD8+ effector T cells for adoptive cell therapy. Nat. Metab. 2020, 2, 703-716. [CrossRef]

56. Wang, T.; Gnanaprakasam, J.N.R.; Chen, X.; Kang, S.; Xu, X.; Sun, H.; Liu, L.; Rodgers, H.; Miller, E.; Cassel, T.A.; et al. Inosine is an alternative carbon source for CD8+-T-cell function under glucose restriction. Nat. Metab. 2020, 2, 635-647. [CrossRef]

57. Nabe, S.; Yamada, T.; Suzuki, J.; Toriyama, K.; Yasuoka, T.; Kuwahara, M.; Shiraishi, A.; Takenaka, K.; Yasukawa, M.; Yamashita, M. Reinforce the antitumor activity of CD $8+$ T cells via glutamine restriction. Cancer Sci. 2018, 109, 3737-3750. [CrossRef]

58. Foskolou, I.P.; Barbieri, L.; Vernet, A.; Bargiela, D.; Cunha, P.P.; Velica, P.; Suh, E.; Pietsch, S.; Matuleviciute, R.; Rundqvist, H.; et al. The $\mathrm{S}$ enantiomer of 2-hydroxyglutarate increases central memory CD8 populations and improves CAR-T therapy outcome. Blood Adv. 2020, 4, 4483-4493. [CrossRef]

59. Cronin, S.J.F.; Seehus, C.; Weidinger, A.; Talbot, S.; Reissig, S.; Seifert, M.; Pierson, Y.; McNeill, E.; Longhi, M.S.; Turnes, B.L.; et al. The metabolite BH4 controls T cell proliferation in autoimmunity and cancer. Nat. Cell Biol. 2018, 563, 564-568. [CrossRef]

60. Bietz, A.; Zhu, H.; Xue, M.; Xu, C. Cholesterol Metabolism in T Cells. Front. Immunol. 2017, 8, 1664. [CrossRef]

61. Yang, W.; Bai, Y.; Xiong, Y.; Zhang, J.; Chen, S.; Zheng, X.; Meng, X.; Li, L.; Wang, J.; Xu, C.; et al. Potentiating the antitumour response of CD8+ T cells by modulating cholesterol metabolism. Nat. Cell Biol. 2016, 531, 651-655. [CrossRef]

62. Zhao, L.; Li, J.; Liu, Y.; Kang, L.; Chen, H.; Jin, Y.; Zhao, F.; Feng, J.; Fang, C.; Zhu, B.; et al. Cholesterol Esterification Enzyme Inhibition Enhances Antitumor Effects of Human Chimeric Antigen Receptors Modified T Cells. J. Immunother. 2018, 41, 45-52. [CrossRef]

63. Jafarzadeh, L.; Masoumi, E.; Fallah-Mehrjardi, K.; Mirzaei, H.R.; Hadjati, J. Prolonged Persistence of Chimeric Antigen Receptor (CAR) T Cell in Adoptive Cancer Immunotherapy: Challenges and Ways Forward. Front. Immunol. 2020, 11, 702. [CrossRef] [PubMed]

64. Klebanoff, C.A.; Crompton, J.G.; Leonardi, A.J.; Yamamoto, T.N.; Chandran, S.S.; Eil, R.L.; Sukumar, M.; Vodnala, S.K.; Madhusudhanan, S.; Ji, Y.; et al. Inhibition of AKT signaling uncouples T cell differentiation from expansion for receptor-engineered adoptive immunotherapy. JCI Insight 2017, 2. [CrossRef]

65. Yang, J.; Nie, J.; Ma, X.; Wei, Y.; Peng, Y.; Wei, X. Targeting PI3K in cancer: Mechanisms and advances in clinical trials. Mol. Cancer 2019, 18, 1-28. [CrossRef]

66. McNamara, C.R.; Degterev, A. Small-molecule inhibitors of the PI3K signaling network. Future Med. Chem. 2011, 3, 549-565. [CrossRef]

67. Menk, A.V.; Scharping, N.E.; Rivadeneira, D.B.; Calderon, M.J.; Watson, M.J.; Dunstane, D.; Watkins, S.C.; Delgoffe, G.M. 4-1BB costimulation induces $\mathrm{T}$ cell mitochondrial function and biogenesis enabling cancer immunotherapeutic responses. J. Exp. Med. 2018, 215, 1091-1100. [CrossRef]

68. Al-Mansour, M.; Al-Foheidi, M.; Ibrahim, E. Efficacy and safety of second-generation CAR T-cell therapy in diffuse large B-cell lymphoma: A meta-analysis. Mol. Clin. Oncol. 2020, 13, 1-14. [CrossRef] 